\title{
Successful Osimertinib Rechallenge with Steroid Therapy after Osimertinib-induced Interstitial Lung Disease
}

\author{
Tatsunori Kiriu, Daisuke Tamura, Motoko Tachihara, Reina Sekiya, Daisuke Hazama, \\ Masahiro Katsurada, Kyosuke Nakata, Tatsuya Nagano, Masatsugu Yamamoto, \\ Hiroshi Kamiryo, Kazuyuki Kobayashi and Yoshihiro Nishimura
}

\begin{abstract}
:
A 62-year-old male with lung adenocarcinoma harboring an exon 19 deletion in the Epidermal growth factor receptor (EGFR) was treated with EGFR-tyrosine kinase inhibitors (TKIs) and several cytotoxic agents. After administering a fifth-line chemotherapy regimen, a liver biopsy revealed a diagnosis of recurrence with a T790M mutation. After an 82-day course of osimertinib therapy, the patient developed osimertinib-induced interstitial lung disease (ILD). Osimertinib was discontinued, and oral prednisolone was started. The ILD quickly improved, but liver metastases progressed and osimertinib was restarted concurrently with prednisolone. The patient showed neither disease progression nor a recurrence of ILD at 5 months. In situations in which no alternative treatment is available, osimertinib rechallenge should thus be considered as an alternative treatment.
\end{abstract}

Key words: osimertinib, interstitial lung disease (ILD), bronchoalveolar lavage (BAL), transbronchial lung biopsy (TBLB)

(Intern Med 57: 91-95, 2018)

(DOI: 10.2169/internalmedicine.8947-17)

\section{Introduction}

Epidermal growth factor receptor tyrosine kinase inhibitors (EGFR-TKIs) are the standard treatment for non-small cell lung cancer (NSCLC) patients harboring mutations of the EGFR. Third-generation EGFR-TKI osimertinib is a potent inhibitor of active EGFR mutations (such as exon 19 deletion, exon $21 \mathrm{~L} 858 \mathrm{R}$ point mutation) and a T790M mutation. However, the most serious adverse reaction associated with treatment by EGFR-TKIs is drug-induced interstitial lung disease (ILD). A case of EGFR mutation-harboring NSCLC that was successfully retreated with osimertinib after the patient developed osimertinib-induced ILD is described.

\section{Case Report}

A 62-year-old Japanese male with a 30 pack-year smok- ing history was diagnosed with stage IV lung adenocarcinoma (cT2aN3M1b) with bone metastases. On examination of a lung biopsy specimen, a diagnosis of adenocarcinoma with the presence of an exon 19 deletion in the EGFR was made. He received up to fifth-line chemotherapy (erlotinib as first-line chemotherapy, cisplatin plus pemetrexed as second-line, carboplatin plus nanoparticle albumin-bound paclitaxel as third-line, afatinib as fourth-line, docetaxel plus bevacizumab as fifth-line), and the evaluation after 6 courses of docetaxel plus bevacizumab showed disease progression, mainly with liver metastases. Since he still maintained a good performance status, a liver biopsy was performed to check for the presence of a T790M mutation. On examination of the liver biopsy specimen, adenocarcinoma with an exon 19 deletion and a T790M mutation was diagnosed.

Osimertinib (80 mg daily) was administered as a sixthline chemotherapy. Before the administration of osimertinib, chest high-resolution computed tomography (CT) showed no interstitial change in the lung field. However, after an 82-

Division of Respiratory Medicine, Department of Internal Medicine, Kobe University Graduate School of Medicine, Japan Received: January 31, 2017; Accepted: May 14, 2017; Advance Publication by J-STAGE: October 16, 2017 Correspondence to Dr. Daisuke Tamura, dtamura@med.kobe-u.ac.jp 

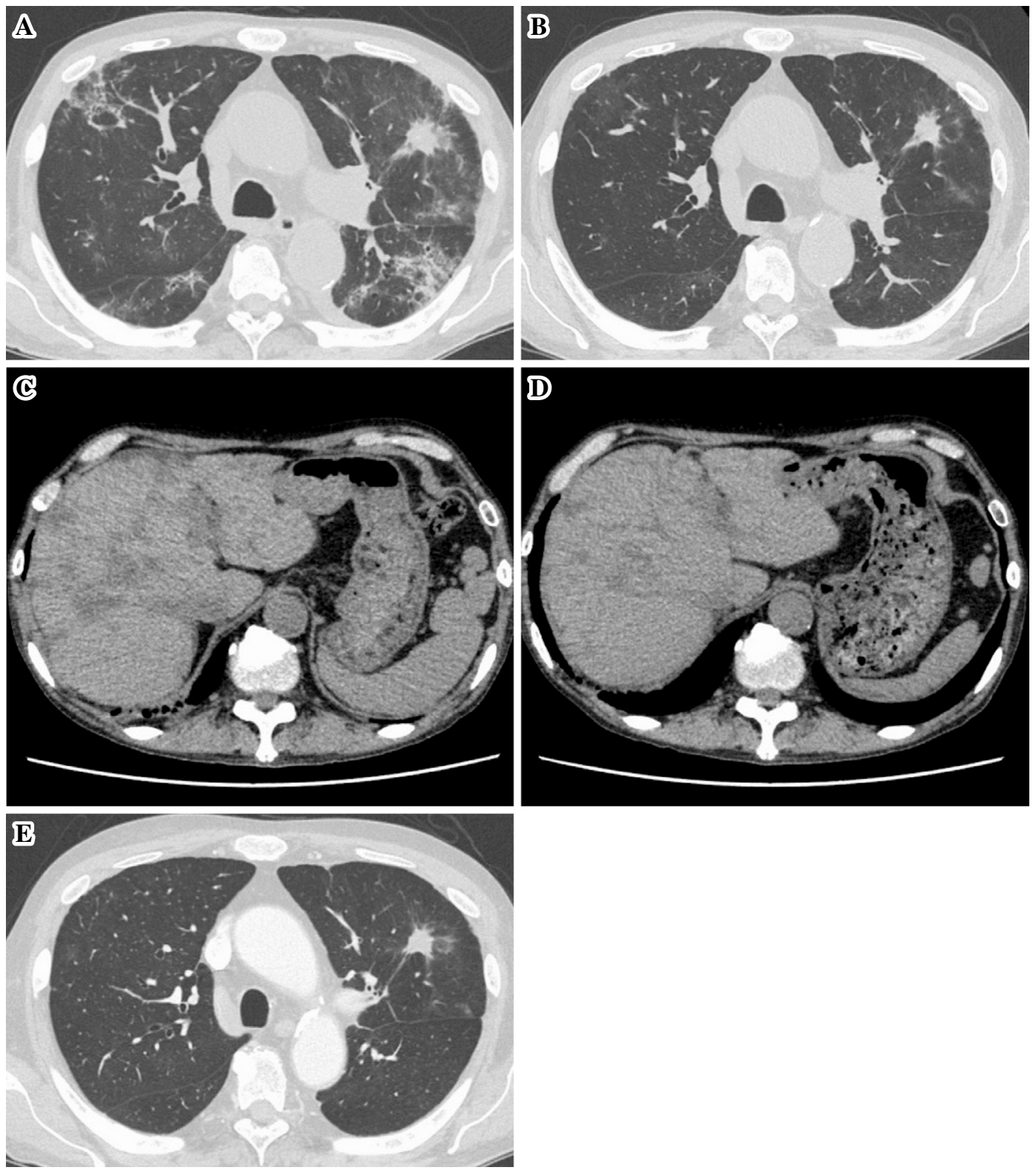

Figure 1. A chest computed tomography scan. (A) 82 days after the initiation of osimertinib. The scan shows bilateral multiple ground-glass opacities (GGOs). (B) One and a half months after the discontinuation of osimertinib. The scan shows an improvement of GGOs and (C) an enlargement of liver metastases. (D) Two weeks after osimertinib retreatment in combination with steroid therapy. Liver metastases have improved. (E) Three months after osimertinib retreatment in combination with steroid therapy. The primary lesion shows little change in size, and no recurrence of ILD is observed.

day course of osimertinib therapy, the patient complained of cough. Chest CT showed bilateral multiple ground-glass opacities (GGOs), despite the fact that the lung cancer (mainly liver metastases) appeared to have improved (Fig. 1A). Osimertinib-induced ILD was suspected, and the administration of osimertinib was discontinued immediately. The patient's laboratory values on admission were as follows: hemoglobin $13.0 \mathrm{~g} / \mathrm{dL}$ (reference range, 13.6-17.0 g/ $\mathrm{dL}$ ), white-cell count $6,100 / \mathrm{mm}^{3}$ (reference range, 4,000$8,500 / \mathrm{mm}^{3}$ ), platelet count $116,000 / \mathrm{mm}^{3}$ (reference range,

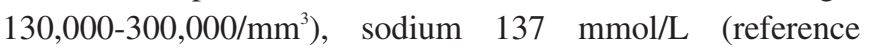
range, $137-146 \mathrm{mmol} / \mathrm{L}$ ), potassium $4.4 \mathrm{mmol} / \mathrm{L}$ (reference range, 3.5-4.7 $\mathrm{mmol} / \mathrm{L}$ ), chloride $106 \mathrm{mmol} / \mathrm{L}$ (reference range, 99-109 mmol/L), blood urea nitrogen $10.6 \mathrm{mg} / \mathrm{dL}$ (reference range, $9-22 \mathrm{mg} / \mathrm{dL}$ ), creatinine $0.92 \mathrm{mg} / \mathrm{dL}$ (reference range, $0.5-1.3 \mathrm{mg} / \mathrm{dL}$ ), aspartate aminotransferase 36 $\mathrm{U} / \mathrm{L}$ (reference range, 13-31 U/L), alanine aminotransferase $33 \mathrm{U} / \mathrm{L}$ (reference range, 8-34 U/L), lactate dehydrogenase $244 \mathrm{U} / \mathrm{L}$ (reference range, 115-217 U/L), total bilirubin 0.6 $\mathrm{mg} / \mathrm{dL}$ (reference range, $0.3-1.0 \mathrm{mg} / \mathrm{dL}$ ), C-reactive protein $0.31 \mathrm{mg} / \mathrm{dL}$ (reference range, $0-0.3 \mathrm{mg} / \mathrm{dL}$ ) and albumin 3.6 $\mathrm{g} / \mathrm{dL}$ (reference range, 4.1-5.0 g/dL). The concentrations of serum krebs von den Lungen-6 (KL-6) and surfactant protein-D (SP-D), markers for lung epithelial injury, were 


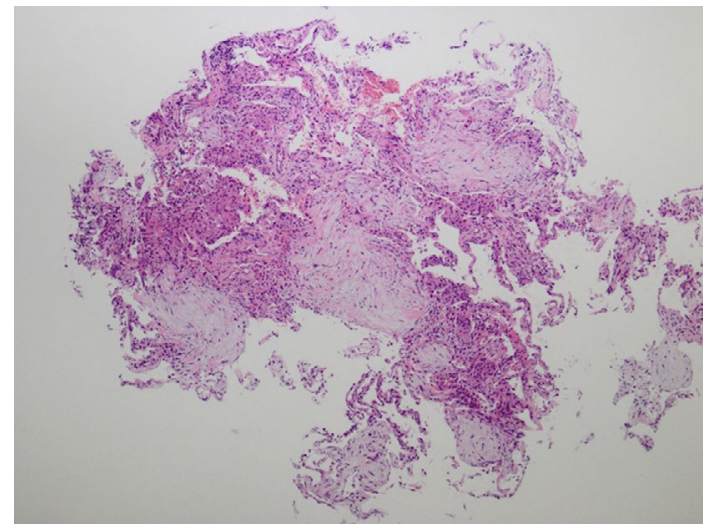

Figure 2. A transbronchial lung biopsy specimen, Hematoxylin and Eosin staining, low-power field $(\times 40)$. Granulation tissue indicating a pattern of organizing pneumonia is observed.

$725 \mathrm{U} / \mathrm{mL}$ (reference range, $0-500 \mathrm{U} / \mathrm{mL}$ ) and $35.1 \mathrm{ng} / \mathrm{mL}$ (reference range, 0-110 U/mL), respectively. We did not measure beta-D-glucan or cytomegalovirus pp65 antigen (C7-HRP). Bronchoalveolar lavage (BAL) and transbronchial lung biopsy (TBLB) of the left upper lobe were performed. The recovery rate was $61 \%(91 / 150 \mathrm{~mL})$. A BAL fluid (BALF) analysis demonstrated an elevated total cell count $\left(58 \times 10^{5} / \mathrm{mL}\right)$, with $32 \%$ lymphocytes. The CD4+/8+ lymphocyte ratio was 0.12 . A few neutrophils and eosinophils were detected. There was no evidence of cytomegalovirus (CMV)-infected cells on BALF cytology. Grocott staining was performed on the BALF, but there was no fungus, including Pneumocystis jirovecii. In addition, no bacteria or acid-fast pathogens were detected in the BALF. Transbronchial lung biopsy specimens showed a strong infiltration of CD8-positive T-cell lymphocytes and a weak infiltration of CD4-positive helper T-cell lymphocytes, mainly in the alveolar septa. This pathological pattern was mainly lymphocytic alveolitis with partial organization in several alveolar spaces, and it was similar to that of a previous report (Fig. 2) (1). Based on these findings, the ground-glass shadows were considered to be consistent with osimertinibinduced ILD. The patient was given oral prednisolone (PSL; $0.5 \mathrm{mg}$ per $\mathrm{kg}, 35 \mathrm{mg}$ daily) with a discontinuation of osimertinib under careful observation. The steroid therapy was tapered from $35 \mathrm{mg}$ to $25 \mathrm{mg}$ daily 2 weeks later, and no enlargement of the interstitial shadows was observed (Fig. 1B). However, liver metastases progressed again (Fig. 1C) and hepatic enzymes were elevated one and a half months after the discontinuation of osimertinib [aspartate aminotransferase (AST) 81 U/L, reference range, 13-31 U/L; alanine aminotransferase (ALT) $169 \mathrm{U} / \mathrm{L}$, reference range 8$34 \mathrm{U} / \mathrm{L}]$. He and his family strongly requested a rechallenge of osimertinib despite full knowledge of the risk of ILD recurrence. Since no alternative treatment for lung cancer was available, the patient was given detailed information about the potential fatal risk of ILD recurrence and was administered osimertinib (40 mg daily, half of the standard initial dose) concurrently with oral PSL (25 mg daily). After 2 weeks, the hepatic enzymes normalized and the liver metastases improved without ILD recurrence (Fig. 1D). At the time of this writing, the patient had shown neither disease progression nor any recurrence of ILD for 5 months (Fig. 1E).

\section{Discussion}

To the best of our knowledge, this is the first case report of osimertinib-induced ILD in which a bronchoscopic examination was performed and in which retreatment with osimertinib after developing drug-induced ILD was successfully carried out.

Osimertinib is effective against active EGFR mutations and a T790M mutation and it has recently been approved in Japan. In the AURA2 study (a multicenter, open-label, single-arm, phase 2 study), ILD/pneumonitis occurred in $2 \%$ and it was fatal in $0.5 \%$ of 211 patients (2). This is less than or equivalent to the previous result for EGFR-TKI-induced ILD (3-5). In an imaging study of 70 patients, the average mortality rate of gefitinib-induced ILD was reported to be $44.3 \%$, whereas that of 20 patients with a diffuse alveolar damage (DAD) pattern was 75\% (6). Transbronchial lung biopsy specimens of the present patient showed an organizing pneumonia pattern (non-DAD pattern), so a better prognosis could be predicted from this perspective. Moreover, Mamesaya et al. reported that osimertinib-induced ILD pathologically displayed an organizing pneumonia pattern, and this is the only report of the pathology related to osimertinib at present. The present case displayed a similar pathological pattern, and more pathological reports about osimertinibrelated ILD are therefore needed (1).

In literature reviews of EGFR-TKI rechallenge after ILD, rechallenges with steroid therapy never produced a recurrence of ILD, while recurrence was observed without steroid therapy $(7,8)$. Some reported that the combination with steroid therapy might be effective with an EGFR-TKI rechallenge, because an allergic or immunological reaction might induce ILD (9). The present patient also did not experience an ILD exacerbation with osimertinib rechallenge in combination with steroid therapy.

However, there is no definite evidence that the development of EGFR-TKI-induced ILD is drug dose-dependent or an allergic reaction to EGFR-TKIs. There is an opinion that EGFR-TKI dose-reduction plays only a limited role in preventing the recurrence of ILD (7). In clinical trials, retreatment with osimertinib after osimertinib-induced ILD was not allowed (2), and therefore the dosage of osimertinib retreatment has not yet been considered. Meanwhile, we have two ways perform a rechallenge of EGFR-TKI, namely the resumption of the same EGFR-TKI or switching to other EGFR-TKIs. In addition to our case, our literature review shows a total of 16 cases of EGFR-TKI rechallenge (Table) $(7,8,10-18)$. Regarding the resumption cases, recurrences of ILD were observed in 2 cases without a dose reduction of EGFR-TKIs, whereas there was no recurrence of 
Table. Literature Review of EGFR-TKI Rechallenges.

\begin{tabular}{|c|c|c|c|c|c|c|c|}
\hline \multicolumn{8}{|c|}{ EGFR-TKI rechallenge - resumption cases } \\
\hline Cases & Age/gender & Histology & Cause of ILD(dose) & Rechallenge(dose) & $\begin{array}{c}\text { Corticosteroid } \\
\text { during rechallenge }\end{array}$ & $\begin{array}{l}\text { Recurrence } \\
\text { of ILD }\end{array}$ & References \\
\hline 2 & $59 / \mathrm{M}$ & Ad & Gefitinib (250 mg/day) & Gefitinib (250 mg/day) & No & Yes & {$[11]$} \\
\hline 3 & $77 / \mathrm{F}$ & Ad & Erlotinib (150 mg/day) & Erlotinib (100 mg/day) & Yes $\rightarrow$ off & No & {$[12]$} \\
\hline 4 & $68 / \mathrm{M}$ & Ad & Erlotinib (150 mg/day) & Erlotinib (150 mg/day) & Yes $\rightarrow$ off & No & {$[7]$} \\
\hline 5 & $62 / \mathrm{M}$ & Ad & Gefitinib (250 mg/day) & Gefitinib (250 mg/day) & Yes & No & {$[8]$} \\
\hline 6 & $64 / \mathrm{M}$ & Ad & Gefitinib (250 mg/day) & Gefitinib (250 mg/day) & Yes $\rightarrow$ off & Yes & {$[8]$} \\
\hline 7 & $62 / \mathrm{M}$ & Ad & Osimertinib (80 mg/day) & Osimertinib (40 mg/day) & Yes & No & Present case \\
\hline \multicolumn{8}{|c|}{ EGFR-TKI rechallenge - switching cases } \\
\hline Cases & Age/gender & Histology & Cause of ILD(dose) & Rechallenge(dose) & $\begin{array}{c}\text { Corticosteroid } \\
\text { during rechallenge }\end{array}$ & $\begin{array}{l}\text { Recurrence } \\
\text { of ILD }\end{array}$ & References \\
\hline 1 & $28 / \mathrm{F}$ & Ad & Gefitinib (250 mg/day) & Erlotinib (50 mg/day) & Yes & No & {$[13]$} \\
\hline 2 & $62 / \mathrm{M}$ & Ad & Gefitinib (250 mg/day) & Erlotinib (150 mg/day) & NA & No & {$[14]$} \\
\hline 3 & $62 / \mathrm{M}$ & Ad & Gefitinib (250 mg/day) & Erlotinib (150 mg/day) & Yes & No & {$[15]$} \\
\hline 4 & $77 / \mathrm{F}$ & Ad & Gefitinib (250 mg/day) & Erlotinib (100 mg/day) & Yes & No & {$[15]$} \\
\hline 5 & $41 / \mathrm{F}$ & Ad & Gefitinib (250 mg/day) & Erlotinib (75 mg/day) & Yes $\rightarrow$ off & No & {$[16]$} \\
\hline 6 & $62 / \mathrm{F}$ & Ad & Gefitinib (250 mg/day) & Erlotinib (25 mg/day) & No & Yes & {$[17]$} \\
\hline 7 & $49 / \mathrm{F}$ & Ad & Gefitinib (250 mg/day) & Erlotinib (150 mg/day) & No & No & [18] \\
\hline 8 & $74 / \mathrm{F}$ & Ad & Gefitinib (250 mg/day) & Erlotinib (150 mg/day) & Yes $\rightarrow$ off & No & [8] \\
\hline 9 & $71 / \mathrm{F}$ & Ad & Gefitinib (250 mg/day) & Erlotinib (150 mg/day) & Yes & No & {$[8]$} \\
\hline
\end{tabular}

Ad: Adenocarcinoma, NA: not available

ILD in the dose reduction cases. We think that this fact might be partial evidence to supportour selection of dose reduction in this case.

The appropriate dosage of steroids remains to be discussed. In the present case, $0.5 \mathrm{mg} / \mathrm{kg}$ of PSL was administered, and this is the dosage that is often given for organizing pneumonia. Since there is also little evidence available regarding the appropriate dosage of steroids in a rechallenge with EGFR-TKI after ILD, this point also needs further investigation.

The dosages of both osimertinib and oral PSL were maintained unchanged in the present case, and we have not yet considered whether to change the dose of each drug, because there is little information in the literature regarding the safety, efficacy, appropriate dose of each drug, or any alteration of the dose, as mentioned above. Thus, more similar cases need to be studied.

\section{Conclusion}

Osimertinib rechallenge after osimertinib-induced ILD must be carefully considered based on the balance of a patient's risks and benefits, considering that ILD due to EGFR-TKIs can be life-threatening (4). The present case suggests that osimertinib rechallenge in combination with steroids can be considered after making a careful assessment of the potential risks and benefits in situations in which no alternative treatment is available. In order to assess the safety and the risk of this treatment, more similar cases need to be studied.
The authors state that they have no Conflict of Interest (COI).

\section{References}

1. Mamesaya N, Kenmotsu H, Katsumata M, et al. Osimertinibinduced interstitial lung disease after treatment with anti PD-1 antibody. Invest New Drugs 35: 105-107, 2017.

2. Goss G, Tsai CM, Shepherd FA, et al. Osimertinib for pretreated EGFR Thr790Met-positive advanced non-small-cell lung cancer (AURA2): a multicentre, open-label, single-arm, phase 2 study. Lancet Oncol 17: 1643-1652, 2016.

3. Kudoh S, Kato H, Nishiwaki Y, et al. Interstitial lung disease in Japanese patients with lung cancer. A cohort and nested casecontrol study. Am J Respir Crit Care Med 177: 1348-1357, 2008.

4. Shi L, Tang J, Tong L, Liu Z. Risk of interstitial lung disease with gefitinib and erlotinib in advanced non-small cell lung cancer: A systematic review and meta-analysis of clinical trials. Lung Cancer 83: 231-239, 2014.

5. Ando M, Okamoto I, Yamamoto N, et al. Predictive factors for interstitial lung disease, antitumor response, and survival in nonsmall-cell lung cancer patients treated with gefitinib. J Clin Oncol 24: 2549-2556, 2006.

6. Endo M, Johkoh T, Kimura K, et al. Imaging of gefitinib-related interstitial lung disease: multi-institutional analysis by the West Japan Thoracic Oncology Group. Lung Cancer 52: 135-140, 2006.

7. Togashi Y, Masago K, Hamatani Y, et al. Successful erlotinib rechallenge for leptomeningeal metastases of lung adenocarcinoma after erlotinib-induced interstitial lung disease: A case report and review of the literature. Lung Cancer 77: 464-468, 2012.

8. Kashiwabara K, Semba H, Fujii S, et al. Outcome in advanced non-small cell lung cancer patients with successful rechallenge after recovery from epidermal growth factor receptor tyrosine kinase inhibitor-induced interstitial lung disease. Cancer Chemother Phae- 
macol 79: 705-710, 2017.

9. Camus P, Kudoh S, Ebina M. Interstitial lung disease associated with drug therapy. Br J Cancer 91: S18-S23, 2004.

10. Takamochi K, Suzuki K, Bashar AH, et al. Readministration of gefitinib in a responder after treatment discontinuation due to gefinitib-related interstitial lung disease: a case report. J Med Case Reports 1: 138, 2007.

11. Suzuki M, Asahina H, Konishi J, et al. Reccurent gefitinibinduced interstitial lung disease. Intern Med 47: 533-536, 2008.

12. Dallas JL, Jantz MA, Lightsey JL, et al. Successful erlotinib rechallenge after erlotinib-induced severe interstitial lund disease. J Thorac Oncol 6: 1142-1143, 2011.

13. Fukui T, Otani S, Hataishi R, et al. Successful rechallenge with erlotinib in a patient with EGFR-mutant lung adenocarcinoma who developed gefitinib-related interstitial lung disease. Cancer Chemother Pharmacol 65: 803-806, 2010.

14. Chang SC, Chang CY, Chen CY, et al. Successful erlotinib rechallenge after gefitinib-induced acute interstitial pneumonia. J Thorac Oncol 7: 1105-1106, 2010.
15. Takeda M, Okamoto I, Makimura C, et al. Successful treatment with erlotinib after gefitinib-induced severe interstitial lung disease. J Thorac Oncol 7: 1103-1104, 2010.

16. Tian Q, Chen LA. Erlotinib achieved partial response in a nonsmall cell lung cancer patient with gefitinib-induced interstitial lung disease. Case Rep Oncol 4: 464-466, 2011.

17. Arakawa N, Tsujita A, Saito N, et al. Successful erlotinib rechallenge after both gefitinib- and erlotinib-induced sever interstitial lung disease. Respirology Case Reports 1: 17-19, 2013.

18. Nakamichi S, Kubota K, Horinouchi H, et al. Successful EGFRTKI rechallenge of leptomeningeal carcinomatosis after gefitinibinduced interstitial lung disease. Jpn J Clin Oncol 43: 422-425, 2013.

The Internal Medicine is an Open Access article distributed under the Creative Commons Attribution-NonCommercial-NoDerivatives 4.0 International License. To view the details of this license, please visit (https://creativecommons.org/licenses/ by-nc-nd/4.0/).

(C) 2018 The Japanese Society of Internal Medicine Intern Med 57: 91-95, 2018 